\title{
DIMINISHING THE HEAT TRANSFER HOT SPOT EFFECT IN A HORIZONTAL ANNULUS USING OPTIMAL POSITIONING OF FINS
}

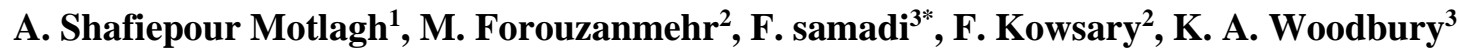

\author{
${ }^{1}$ School of Mechatronics systems Engineering, Simon Fraser University, Burnaby, BC V5A 1S6, Canada \\ ${ }^{2}$ School of Mechanical Engineering, University College of Engineering, University of Tehran, \\ Tehran, Iran \\ ${ }^{3}$ Mechanical Engineering Department, University of Alabama, Tuscaloosa, AL 35401, USA
}

\begin{abstract}
Hot spot in a horizontal annulus is moderated by using a single as well as double fins. The annular space confined between two concentric horizontal cylinders is filled with air and the fins are attached (inward) to the outer cylinder. The internal cylinder is subjected to heat flux boundary condition, and the maximum temperature on its surface (i.e., the hot spot) is taken as the objective function, which is to be minimized by placement of inward fins in the outer surface in an optimal manner. The objective function is minimized using a hybrid Genetic algorithm and Pattern search method with the circumferential locations and the lengths of the fins taken as the design variables.

Optimal positioning of a single fin reduced the hot spot temperature considerably. Adding another fin lowered the hot spot temperature even further. Interestingly, in both cases the heat transfer enhancement and the optimal locations and dimensions of the fins are found to be independent of the Rayleigh number.
\end{abstract}

KEY WORDS: Optimization, Heat transfer enhancement, Fins, Free convection, Genetic algorithm, Pattern search Method, Computational fluid dynamics

\section{INTRODUCTION}

Natural convection heat transfer inside enclosures occurs in a variety of engineering applications. In some of these applications, like cooling of electrical equipment [1], the hot spot temperature limits the overall performance or working life.

Study of natural convection heat transfer between two concentric cylinders goes back to 1930s. Kuehn and Goldstein $[2,3]$ carried experimental and theoretical-numerical studies of natural convection in the annulus between horizontal concentric cylinders. Rahnama and Farhadi [4] studied the effect of radial fins on twodimensional laminar and turbulent natural convection in a horizontal annulus. They used 2 to 12 fins in different configuration. Abu-Nada et al. [5] investigated heat transfer enhancement in concentric annuli using nanofluids. Idrissi et al. [6] utilized isothermal fins of low height to enhance heat transfer in the annular space confined between two concentric cylinders.

In this research, the annulus confined between two concentric cylinders is filled with air. The outer cylinder is fitted with either a single inward-pointing fin or a combination of two such fins to diminish the hot spot effect. Optimal set of design variables including the circumferential locations and lengths of the fins are established by means of an optimization procedure.

\section{PROBLEM FORMULATION}

A schematic depiction of the horizontal annulus is shown in Fig. 1. At first, the two-dimensional domain is solved numerically using conventional methods of CFD. Results of this simulation will be used both as a reference point and as a way to validate the numerical results with previous studies of laminar free convection heat transfer. The inner cylinder is subject to a uniform heat flux boundary condition of $215.14 \mathrm{~W} / \mathrm{m}^{2}$, while 
the outer cylinder is kept at an isothermal condition of $308 \mathrm{~K}$. The fins are made of thermal insulator material and therefore modelled as adiabatic surfaces. The Boussinesq approximation is employed to account for variations of density with temperature. All the other thermophysical properties are assumed to be constant.

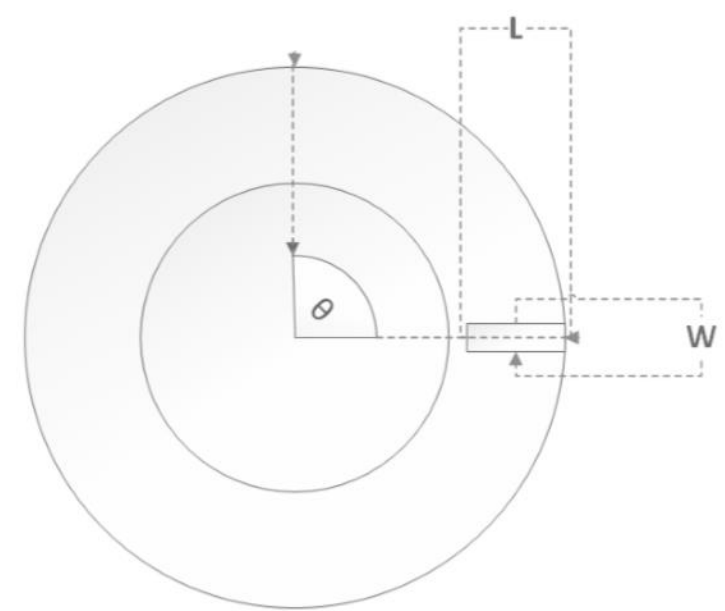

Fig. 1 Schematic of the horizontal annulus with a single fin.

The basic equations governing the fluid flow and heat transfer in cylindrical coordinates are continuity, momentum equations and energy. The computational domain is discretized using a hybrid grid of structured quadrilateral elements near the boundaries and triangular unstructured elements elsewhere. These elements are more refined near the boundaries. The grid independency study is performed to reduce the effect of mesh size on the computational results. Using the experimental correlation provided by Raithby and Hollands [7] for the case of un-finned annulus,

$$
\frac{k_{e f f}}{k}=0.386\left[\frac{P r}{0.861+P r}\right]^{1 / 4} R a_{c}{ }^{1 / 4}
$$

a grid of 38000 elements are chosen. The Numerical results are in good agreement with the imprical correlation presented in Eq. (1) for different Rayleigh numbers in the laminar regime.

The hot spot temperature, i.e. the maximum temperature, on the inner wall of the annulus is taken as the objective function, which is to be minimized. Length $\left(L_{i}\right)$ and circumferential locations of the fins $\left(\theta_{\mathrm{i}}\right)$ constitute the design variables (Fig. 1). A hybrid method of pattern search and genetic algorithm is used to minimize the objective function in an efficient manner without getting stuck in local minimums.

\section{RESULTS}

A flowchart of the solution process is presented in Fig. 2. By fitting a single fin to the horizontal annulus with outer radius of $r_{\mathrm{o}}=0.4 \mathrm{~m}$ and inner radius of $r_{\mathrm{i}}=0.25 \mathrm{~m}$, the hot spot temperature has been reduced to $437.1 \mathrm{~K}$. The optimal set of design variables are listed in the first row of Table 1 and the temperature contours are illustrated in Fig. 3.

Compared to the original hot spot temperature of $452 \mathrm{~K}$ in the un-finned annulus, the optimization algorithm has been able to reduce the hot spot temperature by $15 \mathrm{~K}$.

In the double fin configuration, the hot spot temperature has been diminished by $25 \mathrm{~K}$. The design variables of the optimum point are given in the second row of Table 1. The temperature contours are plotted in Fig. 2. 
Table 1 Optimal values of design variables

\begin{tabular}{lcccc} 
Case & $\mathrm{L}_{1}(\mathrm{~m})$ & $\theta_{1}$ & $\mathrm{~L}_{2}(\mathrm{~m})$ & $\theta_{2}$ \\
\hline Single fin & 0.1413 & 126.2 & - & - \\
Double fin & 0.145 & 45 & 0.145 & 45
\end{tabular}

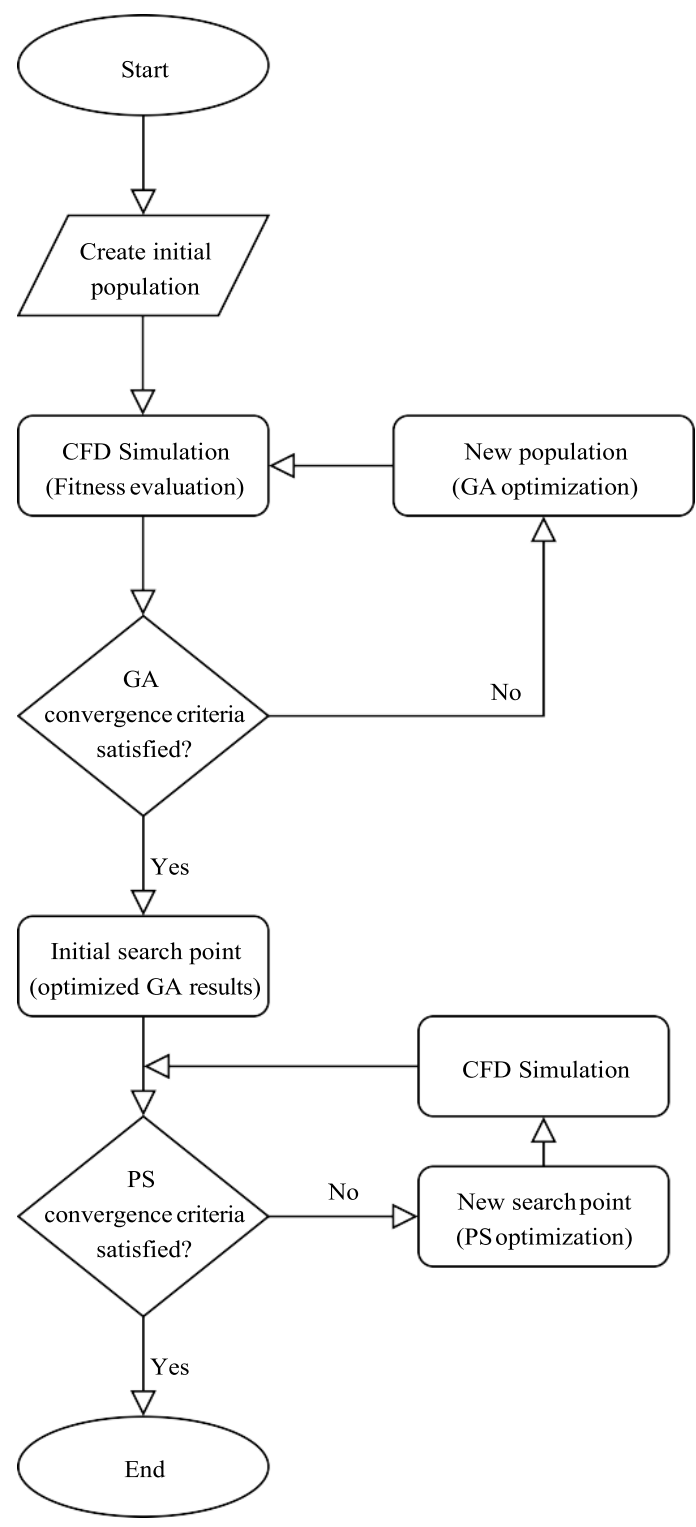

Fig. 2 Flowchart showing the solution process 

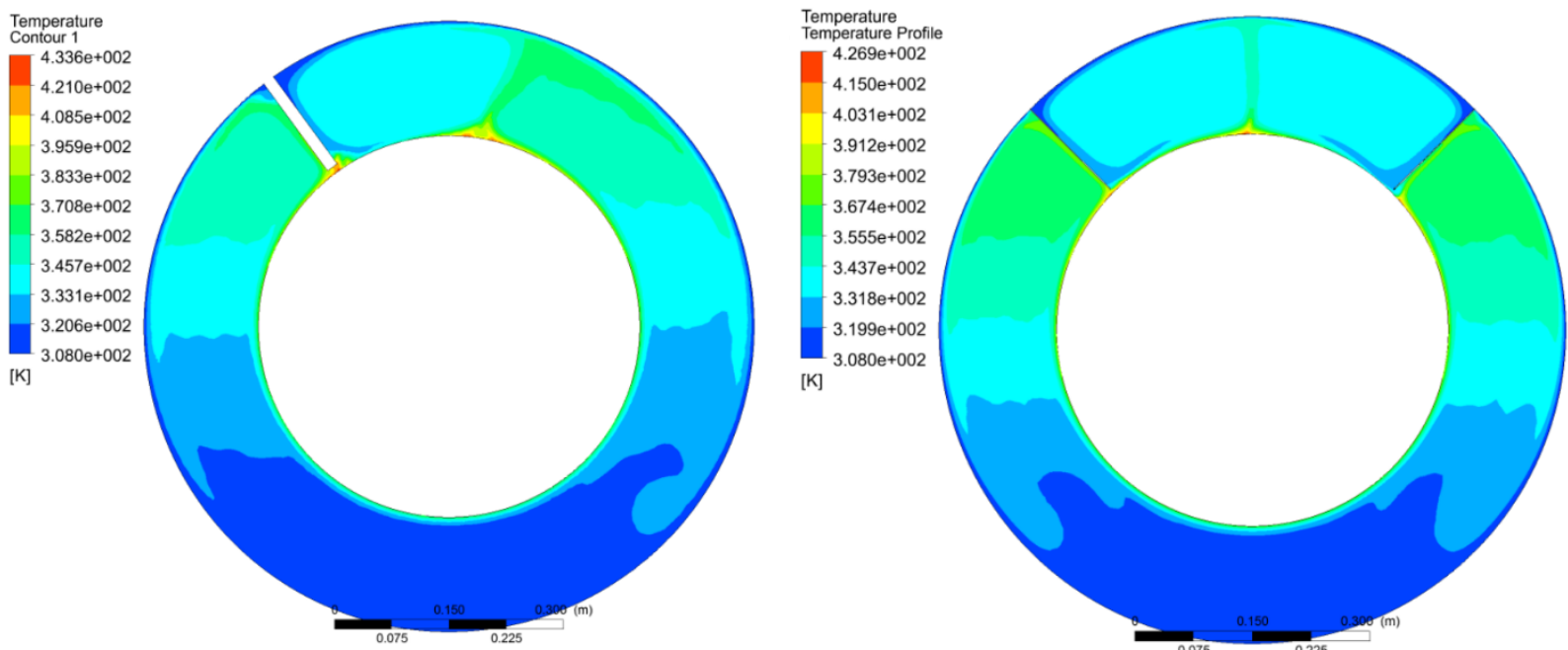

Fig. 3 Temperature contours for the optimal single fin (left), and double fin (right) configuration

\section{CONCLUSIONS}

Fitting a single fin to the annulus diminished the hot spot temperature by $15 \mathrm{~K}$. Adding another fin lowered the hot spot temperature even further. According to Table 1, the optimal lengths of the fins are in close proximity of the upper limit of the applied geometric constraint. This is due to the jet-like effect of the narrow opening between the fin and the inner cylinder that can contribute to further heat transfer enhancement. Using the optimal set of design variables, the Rayleigh number (based on the definition provided by Raithby and Hollands [7]) is calculated as $1.49 \times 10^{6}$. By changing the thermal boundary conditions of the inner and outer cylinders, the optimization process is repeated for different values of the Rayleigh number. Interestingly, in both cases of the single and double fins, the optimal locations and dimensions of the fins are found to be independent of the Rayleigh number for the cases considered in this work.

\section{REFERENCES}

[1] Pedersen, B. O., Doepken, H. C., Bolin, P. C. "Development of a compressed-gas-insulated transmission line." IEEE Transactions on Power Apparatus and Systems, 6, pp 2631-2638, (1971).

[2] Kuehn, T. H., and Goldstein, R. J. "An experimental and theoretical study of natural convection in the annulus between horizontal concentric cylinders.," Journal of Fluid mechanics 74(4), pp. 695-719, (1976).

[3] Kuehn, Thomas H., and Goldstein, R. J. "An experimental study of natural convection heat transfer in concentric and eccentric horizontal cylindrical annuli." Journal of Heat transfer, 100(4), pp. 635-640, (1978).

[4] Rahnama, Mohammad, and Farhadi, Mousa. "Effect of radial fins on two-dimensional turbulent natural convection in a horizontal annulus." International journal of thermal sciences, 43(3), pp. 255-264, (2004).

[5] Abu-Nada, Masoud, E., Z., Hijazi, A. "Natural convection heat transfer enhancement in horizontal concentric annuli using nanofluids." International Communications in Heat and Mass Transfer, 35(5), pp. 657-665, (2008).

[6] Idrissi, Ahmed, Cheddadi, Abdelkhalek, Ouazzani, Mohammed, T., "Heat transfer in an annular space fitted with heating isothermal blocks: Numerical bifurcation for low blocks height." Case studies in thermal engineering, 7, pp.1-7, (2016).

[7] Raithby, G. D., and Hollands, K. G. T., "A General Method of Obtaining Approximate Solutions to Laminar and Turbulent Free Convection Problems," in T. F. Irvine and J. P. Hartnett, Eds., Advances in Heat Transfer, Vol. 11, New York, Academic Press, pp. 265-315, (1975). 